\title{
Measurement of the charged particle multiplicity of weakly decaying $\mathrm{B}$ hadrons
}

DELPHI Collaboration

\begin{abstract}
From the $Z$ decays recorded in 1994 and 1995 by the DELPHI detector at LEP, the charged particle multiplicity of weakly decaying $B$ hadrons was measured to be:

$$
4.97 \pm 0.03 \pm 0.06
$$

excluding the $K^{0}$ and $\Lambda$ decay products.
\end{abstract}


P.Abreu $^{21}$, W.Adam ${ }^{49}$, T.Adye ${ }^{36}$, P.Adzic ${ }^{11}$, I.Ajinenko ${ }^{41}$, G.D.Alekseev ${ }^{16}$, R.Alemany ${ }^{48}$, P.P.Allport ${ }^{22}$, S.Almehed $^{24}$, U.Amaldi ${ }^{9}$, S.Amato ${ }^{46}$, P.Andersson ${ }^{43}$, A.Andreazza ${ }^{9}$, P.Antilogus ${ }^{25}$, W-D.Apel ${ }^{17}$, Y.Arnoud ${ }^{14}$, B.Asman ${ }^{43}$, J-E.Augustin ${ }^{25}$, A.Augustinus ${ }^{9}$, P.Baillon ${ }^{9}$, P.Bambade ${ }^{19}$, F.Barao ${ }^{21}$, R.Barbier ${ }^{25}$, D.Y.Bardin ${ }^{16}$, G.Barker $^{9}$, A.Baroncelli ${ }^{39}$, O.Barring ${ }^{24}$, M.Battaglia ${ }^{15}$, M.Baubillier $^{23}$, K-H.Becks ${ }^{51}$, M.Begalli ${ }^{6}$, P.Beilliere ${ }^{8}$, Yu.Belokopytov ${ }^{9,52}$, A.C.Benvenuti ${ }^{5}$, C.Berat ${ }^{14}$, M.Berggren ${ }^{25}$, D.Bertini ${ }^{25}$, D.Bertrand ${ }^{2}$, M.Besancon ${ }^{38}$, F.Bianchi ${ }^{44}$, M.Bigi ${ }^{44}$, M.S.Bilenky ${ }^{16}$, M-A.Bizouard ${ }^{19}$, D.Bloch ${ }^{10}$, M.Bonesini ${ }^{27}$, W.Bonivento ${ }^{27}$, M.Boonekamp ${ }^{38}$, P.S.L.Booth ${ }^{22}$, A.W.Borgland ${ }^{4}$, G.Borisov ${ }^{38}$, C.Bosio ${ }^{39}$, O.Botner ${ }^{47}$, E.Boudinov ${ }^{30}$, B.Bouquet $^{19}$, C.Bourdarios ${ }^{19}$, T.J.V.Bowcock ${ }^{22}$, I.Boyko ${ }^{16}$, I.Bozovic ${ }^{11}, \quad$ M.Bozzo ${ }^{13}$, P.Branchini ${ }^{39}$, K.D.Brand ${ }^{35}$, T.Brenke ${ }^{51}$, R.A.Brenner ${ }^{47}$, R.Brown ${ }^{9}$, P.Bruckman ${ }^{35}$, J-M.Brunet ${ }^{8}$, L.Bugge ${ }^{32}$, T.Buran ${ }^{32}$, T.Burgsmueller ${ }^{51}$, P.Buschmann ${ }^{51}$, S.Cabrera ${ }^{48}$, M.Caccia $^{27}$, M.Calvi $^{27}$, A.J.Camacho Rozas ${ }^{40}$, T.Camporesi ${ }^{9}$,

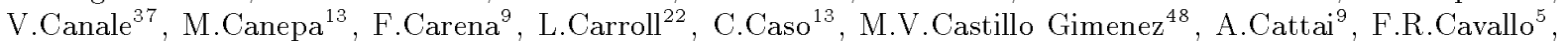
Ch.Cerruti $^{10}$, V.Chabaud ${ }^{9}$, M.Chapkin ${ }^{41}$, Ph.Charpentier ${ }^{9}$, L.Chaussard ${ }^{25}$, P.Checchia ${ }^{35}$, G.A.Chelkov ${ }^{16}$, M.Chen $^{2}$, R.Chierici ${ }^{44}$, P.Chliapnikov ${ }^{41}$, P.Chochula ${ }^{7}$, V.Chorowicz ${ }^{25}$, J.Chudoba ${ }^{29}$, P.Collins ${ }^{9}$, M.Colomer ${ }^{48}$, R.Contri ${ }^{13}$, E.Cortina ${ }^{48}$, G.Cosme $^{19}$, F.Cossutti ${ }^{38}$, J-H.Cowell ${ }^{22}$, H.B.Crawley ${ }^{1}$, D.Crennell ${ }^{36}$, G.Crosetti ${ }^{13}$, J.Cuevas Maestro ${ }^{33}$, S.Czellar ${ }^{15}$, B.Dalmagne ${ }^{19}$, G.Damgaard ${ }^{28}$, M.Davenport ${ }^{9}$, W.Da Silva ${ }^{23}$, A.Deghorain ${ }^{2}$, G.Della Ricca ${ }^{45}$, P.Delpierre ${ }^{26}$, N.Demaria ${ }^{9}$, A.De Angelis ${ }^{9}$, W.De Boer ${ }^{17}$, S.De Brabandere ${ }^{2}$, C.De Clercq ${ }^{2}$, B.De Lotto ${ }^{45}$, A.De Min ${ }^{35}$, L.De Paula ${ }^{46}$, H.Dijkstra ${ }^{9}$, L.Di Ciaccio ${ }^{37}$, A.Di Diodato ${ }^{37}$, A.Djannati ${ }^{8}$, J.Dolbeau $^{8}$, K.Doroba ${ }^{50}$, M.Dracos ${ }^{10}$, J.Drees ${ }^{51}$, K.-A.Drees ${ }^{51}$, M.Dris ${ }^{31}$, A.Duperrin ${ }^{25}$, J-D.Durand ${ }^{25,9}$, R.Ehret $^{17}$, G.Eigen ${ }^{4}$, T.Ekelof ${ }^{47}, \quad$ G.Ekspong ${ }^{43}$, M.Ellert ${ }^{47}, \quad$ M.Elsing ${ }^{9}$, J-P.Engel ${ }^{10}, \quad$ B.Erzen $^{42}$, M.Espirito Santo ${ }^{21}$, E.Falk ${ }^{24}$, G.Fanourakis ${ }^{11}$, D.Fassouliotis ${ }^{11}$, J.Fayot ${ }^{23}$, M.Feindt ${ }^{17}$, P.Ferrari ${ }^{27}$, A.Ferrer ${ }^{48}$, S.Fichet $^{23}$, A.Firestone ${ }^{1}$, P.-A.Fischer ${ }^{9}$, U.Flagmeyer ${ }^{51}$, H.Foeth ${ }^{9}$, E.Fokitis ${ }^{31}$, F.Fontanelli3 ${ }^{13}$, B.Franek ${ }^{36}$, A.G.Frodesen ${ }^{4}$, R.Fruhwirth ${ }^{49}$, F.Fulda-Quenzer ${ }^{19}$, J.Fuster ${ }^{48}$, A.Galloni ${ }^{22}$, D.Gamba ${ }^{44}$, M.Gandelman ${ }^{46}$, C.Garcia $^{48}$, J.Garcia ${ }^{40}$, C.Gaspar ${ }^{9}$, M.Gaspar ${ }^{46}$, U.Gasparini ${ }^{35}$, Ph.Gavillet ${ }^{9}$, E.N.Gazis ${ }^{31}$, D.Gele ${ }^{10}$, J-P.Gerber ${ }^{10}$, L.Gerdyukov ${ }^{41}$, N.Ghodbane ${ }^{25}$, I.Gil ${ }^{48}$, F.Glege ${ }^{51}$, R.Gokieli ${ }^{50}$, B.Golob ${ }^{42}$, P.Goncalves ${ }^{21}$, I.Gonzalez Caballero ${ }^{40}$, G.Gopal ${ }^{36}$, L.Gorn ${ }^{1,53}$, M.Gorski ${ }^{50}$, Yu.Gouz ${ }^{41}$, V.Gracco ${ }^{13}$, J.Grahl ${ }^{1}$, E.Graziani ${ }^{39}$, C.Green ${ }^{22}$, A.Grefrath ${ }^{51}$, P.Gris ${ }^{38}$, G.Grosdidier ${ }^{19}$, K.Grzelak ${ }^{50}$, M.Gunther ${ }^{47}$, J.Guy ${ }^{36}$, F.Hahn ${ }^{9}$, S.Hahn ${ }^{51}$, S.Haider $^{9}$, A.Hallgren ${ }^{47}$, K.Hamacher ${ }^{51}$, F.J.Harris ${ }^{34}$, V.Hedberg ${ }^{24}$, S.Heising ${ }^{17}$, R.Henriques ${ }^{21}$, J.J.Hernandez ${ }^{48}$, P.Herquet ${ }^{2}$, H.Herr ${ }^{9}$, T.L.Hessing ${ }^{34}$, J.-M.Heuser ${ }^{51}$, E.Higon $^{48}$, S-O.Holmgren ${ }^{43}$, P.J.Holt ${ }^{34}$, D.Holthuizen $^{30}$, S.Hoorelbeke ${ }^{2}$, M.Houlden ${ }^{22}$, J.Hrubec ${ }^{49}$, K.Huet ${ }^{2}$, K.Hultqvist ${ }^{43}$, J.N.Jackson ${ }^{22}$ ', R.Jacobsson ${ }^{43}$, P.Jalocha ${ }^{9}$, R.Janik ${ }^{7}$, Ch.Jarlskog ${ }^{24}$, G.Jarlskog ${ }^{24}$, P.Jarry ${ }^{38}$, B.Jean-Marie ${ }^{19}$, E.K.Johansson ${ }^{43}$, L.Jonsson ${ }^{24}$, P.Jonsson ${ }^{24}$, C.Joram ${ }^{9}$, P.Juillot ${ }^{10}$, F.Kapusta ${ }^{23}$, K.Karafasoulis ${ }^{11}$, S.Katsanevas ${ }^{25}$, E.C.Katsoufis $^{31}$, R.Keranen ${ }^{4}$, Yu.Khokhlov ${ }^{41}$, B.A.Khomenko ${ }^{16}$, N.N.Khovanski ${ }^{16}$, B.King ${ }^{22}$, N.J.Kjaer ${ }^{30}$, O.Klapp ${ }^{51}$, H.Klein ${ }^{9}$, P.Kluit ${ }^{30}$, D.Knoblauch ${ }^{17}$, P.Kokkinias ${ }^{11}$, M.Koratzinos ${ }^{9}$, V.Kostioukhine ${ }^{41}$, C.Kourkoumelis ${ }^{3}$, O.Kouznetsov ${ }^{16}$, M.Krammer ${ }^{49}$, C.Kreuter ${ }^{9}$, I.Kronkvist ${ }^{24}$, J.Krstic ${ }^{11}$, Z.Krumstein ${ }^{16}$, P.Kubinec $^{7}$, W.Kucewicz ${ }^{18}$, K.Kurvinen ${ }^{15}$, C.Lacasta ${ }^{9}$, J.W.Lamsa ${ }^{1}$, L.Lanceri ${ }^{45}$, D.W.Lane ${ }^{1}$, P.Langefeld ${ }^{51}$, V.Lapin ${ }^{41}$, J-P.Laugier ${ }^{38}$, R.Lauhakangas ${ }^{15}$, G.Leder ${ }^{49}$, F.Ledroit ${ }^{14}$, V.Lefebure ${ }^{2}$, L.Leinonen ${ }^{43}$, A.Leisos ${ }^{11}$, R.Leitner $^{29}$, J.Lemonne ${ }^{2}$, G.Lenzen ${ }^{51}$, V.Lepeltier ${ }^{19}$, T.Lesiak ${ }^{18}$, M.Lethuillier $^{38}$, J.Libby ${ }^{34}$, D.Liko ${ }^{9}$, A.Lipniacka $^{43}$, I.Lippi $^{35}$, B.Loerstad ${ }^{24}$, J.G.Loken ${ }^{34}$, J.H.Lopes ${ }^{46}$, J.M.Lopez $^{40}$, D.Loukas ${ }^{11}$, P.Lutz $^{38}$, L.Lyons $^{34}$, J.MacNaughton ${ }^{49}$, J.R.Mahon ${ }^{6}$, A.Maio ${ }^{21}$, A.Malek ${ }^{51}$, T.G.M.Malmgren ${ }^{43}$, V.Malychev ${ }^{16}$, J.Marco $^{40}$, R.Marco ${ }^{40}$, B.Marechal ${ }^{46}$, M.Margoni ${ }^{35}$, J-C.Marin ${ }^{9}$, C.Mariotti ${ }^{9}$, A.Markou ${ }^{11}$, C.Martinez-Rivero $^{33}$, F.Martinez-Vidal ${ }^{48}$, S.Marti i Garcia ${ }^{22}$, F.Matorras ${ }^{40}$, C.Matteuzzi ${ }^{27}$, G.Matthiae ${ }^{37}$, F.Mazzucato $^{35}$, M.Mazzucato ${ }^{35}$, M.Mc Cubbin ${ }^{22}$, R.Mc Kay ${ }^{1}$, R.Mc Nulty ${ }^{9}$, G.Mc Pherson ${ }^{22}$, J.Medbo ${ }^{47}$, C.Meroni $^{27}$, M.Michelotto ${ }^{35}$, E.Migliore ${ }^{44}$, L.Mirabito ${ }^{25}$, W.A.Mitaroff ${ }^{49}$, U.Mjoernmark ${ }^{24}$, T.Moa ${ }^{43}$, R.Moeller $^{28}$, K.Moenig ${ }^{9}$, M.R.Monge ${ }^{13}$, X.Moreau ${ }^{23}$, P.Morettini ${ }^{13}$, G.Morton ${ }^{34}$, K.Muenich ${ }^{51}$, M.Mulders ${ }^{30}$, L.M.Mundim ${ }^{6}$, W.J.Murray ${ }^{36}$, B.Muryn ${ }^{14,18}$, G.Myatt ${ }^{34}$, T.Myklebust ${ }^{32}$, F.Naraghi ${ }^{14}$, F.L.Navarria ${ }^{5}$, S.Navas $^{48}$, K.Nawrocki ${ }^{50}$, P.Negri ${ }^{27}$, S.Nemecek ${ }^{12}$, N.Neufeld ${ }^{9}$, W.Neumann ${ }^{51}$, N.Neumeister ${ }^{49}$, R.Nicolaidou ${ }^{14}$, B.S.Nielsen ${ }^{28}$, M.Nieuwenhuizen ${ }^{30}$, V.Nikolaenko ${ }^{10}$, M.Nikolenko ${ }^{10,16}$, A.Nomerotski ${ }^{35}$, A.Normand ${ }^{22}$, A.Nygren $^{24}$, W.Oberschulte-Beckmann ${ }^{17}$, V.Obraztsov ${ }^{41}$, A.G.Olshevski ${ }^{16}$, A.Onofre ${ }^{21}$, R.Orava ${ }^{15}$, G.Orazi ${ }^{10}$, K.Osterberg $^{15}$, A.Ouraou ${ }^{38}$, P.Paganini ${ }^{19}$, M.Paganoni ${ }^{27}$, S.Paiano ${ }^{5}$, R.Pain ${ }^{23}$, R.Paiva ${ }^{21}$, J.Palacios ${ }^{34}$, H.Palka ${ }^{18}$, Th.D.Papadopoulou ${ }^{31}$, K.Papageorgiou ${ }^{11}$, L.Pape ${ }^{9}$, C.Parkes ${ }^{34}$, F.Parodi ${ }^{13}$, U.Parzefall ${ }^{22}$,

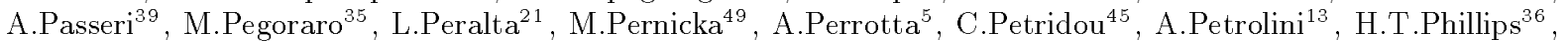
G.Piana $^{13}$, F.Pierre ${ }^{38}$, M.Pimenta ${ }^{21}$, E.Piotto ${ }^{35}$, T.Podobnik ${ }^{42}$, O.Podobrin ${ }^{9}$, M.E.Pol ${ }^{6}$, G.Polok ${ }^{18}$, P.Poropat ${ }^{45}$, V.Pozdniakov ${ }^{16}$, P.Privitera ${ }^{37}$, N.Pukhaeva ${ }^{16}$, A.Pullia ${ }^{27}$, D.Radojicic ${ }^{34}$, S.Ragazzi ${ }^{27}$, H.Rahmani ${ }^{31}$, D.Rakoczy ${ }^{49}$, J.Rames ${ }^{12}$, P.N.Ratoff ${ }^{20}$, A.L.Read ${ }^{32}$, P.Rebecchi ${ }^{9}$, N.G.Redaelli ${ }^{27}$, M.Regler $^{49}$, D.Reid $^{9}$, R.Reinhardt ${ }^{51}$, P.B.Renton ${ }^{34}$, L.K.Resvanis ${ }^{3}$, F.Richard ${ }^{19}$, J.Ridky ${ }^{12}$, G.Rinaudo ${ }^{44}$, O.Rohne ${ }^{32}$, A.Romero $^{44}$, P.Ronchese ${ }^{35}$, E.I.Rosenberg ${ }^{1}$, P.Rosinsky ${ }^{7}$, P.Roudeau ${ }^{19}$, T.Rovelli ${ }^{5}$, V.Ruhlmann-Kleider ${ }^{38}$, A.Ruiz ${ }^{40}$, H.Saarikko ${ }^{15}$, Y.Sacquin ${ }^{38}$, A.Sadovsky ${ }^{16}$, G.Sajot ${ }^{14}$, J.Salt ${ }^{48}$, D.Sampsonidis ${ }^{11}$, M.Sannino ${ }^{13}$, H.Schneider ${ }^{17}$, Ph.Schwemling ${ }^{23}$, U.Schwickerath ${ }^{17}$, M.A.E.Schyns ${ }^{51}$, F.Scuri ${ }^{45}$, P.Seager ${ }^{20}$, Y.Sedykh ${ }^{16}$, A.M.Segar ${ }^{34}$, R.Sekulin ${ }^{36}$, R.C.Shellard ${ }^{6}$, A.Sheridan ${ }^{22}$, R.Silvestre ${ }^{38}$, F.Simonetto ${ }^{35}$, A.N.Sisakian ${ }^{16}$, T.B.Skaali ${ }^{32}$, G.Smadja ${ }^{25}$, N.Smirnov ${ }^{41}, \quad$ O.Smirnova $^{24}$, G.R.Smith ${ }^{36}$, A.Sopczak ${ }^{17}$, R.Sosnowski ${ }^{50}$, D.Souza-Santos ${ }^{6}$, E.Spiriti ${ }^{39}$, P.Sponholz ${ }^{51}$, S.Squarcia ${ }^{13}$, D.Stampfer ${ }^{49}$, C.Stanescu ${ }^{39}$, S.Stanic ${ }^{42}$, S.Stapnes $^{32}$, I.Stavitski $^{35}$, K.Stevenson ${ }^{34}$, A.Stocchi ${ }^{19}$, J.Strauss ${ }^{49}$, R.Strub ${ }^{10}$, B.Stugu ${ }^{4}$, M.Szczekowski ${ }^{50}$, M.Szeptycka ${ }^{50}$, T.Tabarelli $^{27}$, F.Tegenfeldt ${ }^{47}$, F.Terranova ${ }^{27}$, J.Thomas ${ }^{34}$, A.Tilquin ${ }^{26}$, J.Timmermans ${ }^{30}$, L.G.Tkatchev ${ }^{16}$, 
T.Todorov ${ }^{10}$, S.Todorova ${ }^{10}$, D.Z.Toet ${ }^{30}$, A.Tomaradze ${ }^{2}$, B.Tome ${ }^{21}$, A.Tonazzo ${ }^{27}$, L.Tortora ${ }^{39}$, G.Transtromer ${ }^{24}$, D.Treille ${ }^{9}$, G.Tristram ${ }^{8}$, A.Trombini ${ }^{19}$, C.Troncon ${ }^{27}$, A.Tsirou ${ }^{9}$, M-L.Turluer ${ }^{38}$, I.A.Tyapkin ${ }^{16}$, S.Tzamarias $^{11}$, B.Ueberschaer ${ }^{51}$, O.Ullaland ${ }^{9}$, V.Uvarov ${ }^{41}$, G.Valenti ${ }^{5}$, E.Vallazza ${ }^{45}$, C.Vander Velde ${ }^{2}$, G.W.Van Apeldoorn ${ }^{30}$, P.Van Dam ${ }^{30}$, W.K.Van Doninck ${ }^{2}$, J.Van Eldik ${ }^{30}$, A.Van Lysebetten ${ }^{2}$, I.Van Vulpen ${ }^{30}$, N.Vassilopoulos ${ }^{34}$, G.Vegni $^{27}$, L.Ventura ${ }^{35}$, W.Venus ${ }^{36}$, F.Verbeure ${ }^{2}$, M.Verlato ${ }^{35}$, L.S.Vertogradov ${ }^{16}$, V.Verzi ${ }^{37}$, D.Vilanova ${ }^{38}$, L.Vitale $^{45}$, E.Vlasov ${ }^{41}$, A.S.Vodopyanov ${ }^{16}$, V.Vrba ${ }^{12}$, H.Wahlen ${ }^{51}$, C.Walck ${ }^{43}$, C.Weiser ${ }^{17}$, A.M.Wetherell ${ }^{9}$, D.Wicke $^{51}$, J.H.Wickens ${ }^{2}, \quad$ G.R.Wilkinson ${ }^{9}, \quad$ M.Winter ${ }^{10}, \quad$ M.Witek $^{18}$, T.Wlodek ${ }^{19}, \quad$ G.Wolf ${ }^{9}, \quad$ J.Yi $^{1}{ }^{1}$, O.Yushchenko $^{41}$, A.Zalewska ${ }^{18}$, P.Zalewski ${ }^{50}$, D.Zavrtanik ${ }^{42}$, E.Zevgolatakos ${ }^{11}$, N.I.Zimin ${ }^{16}$, G.C.Zucchelli ${ }^{43}$, G.Zumerle ${ }^{35}$

\footnotetext{
${ }^{1}$ Department of Physics and Astronomy, Iowa State University, Ames IA 50011-3160, USA

${ }^{2}$ Physics Department, Univ. Instelling Antwerpen, Universiteitsplein 1, BE-2610 Wilrijk, Belgium and IIHE, ULB-VUB, Pleinlaan 2, BE-1050 Brussels, Belgium

and Faculté des Sciences, Univ. de l'Etat Mons, Av. Maistriau 19, BE-7000 Mons, Belgium

${ }^{3}$ Physics Laboratory, University of Athens, Solonos Str. 104, GR-10680 Athens, Greece

${ }^{4}$ Department of Physics, University of Bergen, Allégaten 55, NO-5007 Bergen, Norway

${ }^{5}$ Dipartimento di Fisica, Università di Bologna and INFN, Via Irnerio 46, IT-40126 Bologna, Italy

${ }^{6}$ Centro Brasileiro de Pesquisas Físicas, rua Xavier Sigaud 150, BR-22290 Rio de Janeiro, Brazil and Depto. de Física, Pont. Univ. Católica, C.P. 38071 BR-22453 Rio de Janeiro, Brazil and Inst. de Física, Univ. Estadual do Rio de Janeiro, rua São Francisco Xavier 524, Rio de Janeiro, Brazil ${ }^{7}$ Comenius University, Faculty of Mathematics and Physics, Mlynska Dolina, SK-84215 Bratislava, Slovakia ${ }^{8}$ Collège de France, Lab. de Physique Corpusculaire, IN2P3-CNRS, FR-75231 Paris Cedex 05, France ${ }^{9} \mathrm{CERN}, \mathrm{CH}-1211$ Geneva 23, Switzerland

${ }^{10}$ Institut de Recherches Subatomiques, IN2P3 - CNRS/ULP - BP20, FR-67037 Strasbourg Cedex, France

${ }^{11}$ Institute of Nuclear Physics, N.C.S.R. Demokritos, P.O. Box 60228, GR-15310 Athens, Greece

${ }^{12}$ FZU, Inst. of Phys. of the C.A.S. High Energy Physics Division, Na Slovance 2, CZ-180 40, Praha 8, Czech Republic

${ }^{13}$ Dipartimento di Fisica, Università di Genova and INFN, Via Dodecaneso 33, IT-16146 Genova, Italy

${ }^{14}$ Institut des Sciences Nucléaires, IN2P3-CNRS, Université de Grenoble 1, FR-38026 Grenoble Cedex, France

${ }^{15}$ Helsinki Institute of Physics, HIP, P.O. Box 9, FT-00014 Helsinki, Finland

${ }^{16}$ Joint Institute for Nuclear Research, Dubna, Head Post Office, P.O. Box 79, RU-101 000 Moscow, Russian Federation

${ }^{17}$ Institut für Experimentelle Kernphysik, Universität Karlsruhe, Postfach 6980, DE-76128 Karlsruhe, Germany

${ }^{18}$ Institute of Nuclear Physics and University of Mining and Metalurgy, Ul. Kawiory 26a, PL-30055 Krakow, Poland

${ }^{19}$ Université de Paris-Sud, Lab. de l'Accélérateur Linéaire, IN2P3-CNRS, Bât. 200, FR-91405 Orsay Cedex, France

${ }^{20}$ School of Physics and Chemistry, University of Lancaster, Lancaster LA1 4YB, UK

${ }^{21}$ LIP, IST, FCUL - Av. Elias Garcia, 14-1 ${ }^{\circ}$, PT-1000 Lisboa Codex, Portugal

${ }^{22}$ Department of Physics, University of Liverpool, P.O. Box 147, Liverpool L69 3BX, UK

${ }^{23}$ LPNHE, IN2P3-CNRS, Univ. Paris VI et VII, Tour 33 (RdC), 4 place Jussieu, FR-75252 Paris Cedex 05, France

${ }^{24}$ Department of Physics, University of Lund, Sölvegatan 14, SE-223 63 Lund, Sweden

${ }^{25}$ Université Claude Bernard de Lyon, IPNL, IN2P3-CNRS, FR-69622 Villeurbanne Cedex, France

26 Univ. d'Aix - Marseille II - CPP. IN2P3-CNRS, FR-13288 Marseille Cedex 09, France

${ }^{27}$ Dipartimento di Fisica, Università di Milano and INFN, Via Celoria 16, IT-20133 Milan, Italy

${ }^{28}$ Niels Bohr Institute, Blegdamsvej 17, DK-2100 Copenhagen $\emptyset$, Denmark

${ }^{29} \mathrm{NC}$, Nuclear Centre of MFF, Charles University, Areal MFF, V Holesovickach 2, CZ-180 00, Praha 8 , Czech Republic

${ }^{30}$ NIKHEF, Postbus 41882, NL-1009 DB Amsterdam, The Netherlands

${ }^{31}$ National Technical University, Physics Department, Zografou Campus, GR-15773 Athens, Greece

${ }^{32}$ Physics Department, University of Oslo, Blindern, NO-1000 Oslo 3, Norway

${ }^{33}$ Dpto. Fisica, Univ. Oviedo, Avda. Calvo Sotelo s/n, ES-33007 Oviedo, Spain

${ }^{34}$ Department of Physics, University of Oxford, Keble Road, Oxford OX1 3RH, UK

${ }^{35}$ Dipartimento di Fisica, Università di Padova and INFN, Via Marzolo 8, IT-35131 Padua, Italy

${ }^{36}$ Rutherford Appleton Laboratory, Chilton, Didcot OX11 OQX, UK

${ }^{37}$ Dipartimento di Fisica, Università di Roma II and INFN, Tor Vergata, IT-00173 Rome, Italy

${ }^{38}$ DAPNIA/Service de Physique des Particules, CEA-Saclay, FR-91191 Gif-sur-Yvette Cedex, France

${ }^{39}$ Istituto Superiore di Sanità, Ist. Naz. di Fisica Nucl. (INFN), Viale Regina Elena 299, IT-00161 Rome, Italy

${ }^{40}$ Instituto de Fisica de Cantabria (CSIC-UC), Avda. los Castros s/n, ES-39006 Santander, Spain

${ }^{41}$ Inst. for High Energy Physics, Serpukov P.O. Box 35, Protvino, (Moscow Region), Russian Federation

42 J. Stefan Institute, Jamova 39, SI-1000 Ljubljana, Slovenia and Department of Astroparticle Physics, School of Environmental Sciences, Kostanjeviska 16a, Nova Gorica, SI-5000 Slovenia, and Department of Physics, University of Ljubljana, SI-1000 Ljubljana, Slovenia

${ }^{43}$ Fysikum, Stockholm University, Box 6730, SE-113 85 Stockholm, Sweden

${ }^{44}$ Dipartimento di Fisica Sperimentale, Università di Torino and INFN, Via P. Giuria 1, IT-10125 Turin, Italy

${ }^{45}$ Dipartimento di Fisica, Università di Trieste and INFN, Via A. Valerio 2, IT-34127 Trieste, Italy and Istituto di Fisica, Università di Udine, IT-33100 Udine, Italy

${ }^{46}$ Univ. Federal do Rio de Janeiro, C.P. 68528 Cidade Univ., Tlha do Fundão BR-21945-970 Rio de Janeiro, Brazil

47 Department of Radiation Sciences, University of Uppsala, P.O. Box 535, SE-751 21 Uppsala, Sweden

${ }^{48}$ IFIC, Valencia-CSIC, and D.F.A.M.N., U. de Valencia, Avda. Dr. Moliner 50, ES-46100 Burjassot (Valencia), Spain

${ }^{49}$ Institut für Hochenergiephysik, Österr. Akad. d. Wissensch., Nikolsdorfergasse 18, AT-1050 Vienna, Austria

${ }^{50}$ Inst. Nuclear Studies and University of Warsaw, Ul. Hoza 69, PL-00681 Warsaw, Poland

${ }^{51}$ Fachbereich Physik, University of Wuppertal, Postfach 100 127, DE-42097 Wuppertal, Germany

52 On leave of absence from IHEP Serpukhov

${ }^{53}$ Now at University of Florida
} 


\section{Introduction}

An important parameter needed in electroweak measurements concerning the $b$ quark is the charged particle multiplicity of weakly decaying B-hadrons, $n_{B}$. As an example, the efficiency to identify a $b \bar{b}$ quarks event depends almost linearly on the value of $n_{B}$. Measurements of the charged particle multiplicities of $B$ hadron decays have been performed previously by the CLEO [1], ARGUS [2], OPAL [3], DELPHI [4] and L3 [5] collaborations. The first two results apply to equally mixed samples of $B_{d}^{0}$ and $B^{+}$mesons, whereas the others concern samples of $B$ hadrons containing all weakly decaying $B$ particles as obtained in the fragmentation of a $b$-quark jet at high energy.

In 1994 and 1995 a large sample of $Z$ decays was collected by the DELPHI experiment with a new Vertex Detector [6] capable of measuring the coordinates of points on tracks in three dimensions, thus improving considerably the $b$-tagging performance. In addition, a major improvement in the reconstruction programs has enhanced the tracking efficiency. The improved $b$ purity allows a better measurement of $n_{B}$.

Two methods of measuring $n_{B}$ are used in this analysis, one based on the sign of the track impact parameters and the other, used only as a cross check, based on reconstructed secondary vertices. The $B$ hadrons have a relative long lifetime $(\approx 1.5 \mathrm{ps})$ and a high mass $(\approx 5 \mathrm{GeV})$, the fragmentation is hard and the decay products have a large value of the impact parameter and originate from well displaced secondary vertices. Particles coming from fragmentation of light quarks instead have an impact parameter distribution centered around zero.

\section{The DELPHI Detector}

The DELPHI detector and its performance have been described in detail in ref. [7]. Here only the new Vertex Detector (VD) [6], the most relevant detector used in this analysis, will be described.

The VD is the innermost detector in DELPHI. It is located between the LEP beam pipe and the Inner Detector. In 1994 the DELPHI Vertex Detector [8] was upgraded to provide a three-dimensional readout [6]. It consists of three concentric layers of silicon microstrip detectors at radii of $6.3,9$ and $11 \mathrm{~cm}$ from the beam line, called the closer, inner and outer layer respectively. The microstrip detectors of the closer and outer layers provide hits in the $R \Phi$ and the $R z$-plane ${ }^{1}$, while for the inner layer only the $R \Phi$ coordinate is measured. For polar angles of $44^{\circ} \leq \theta \leq 136^{\circ}$ a track crosses all the three silicon layers of the VD. The closer layer covers the polar region between $25^{\circ}$ and $155^{\circ}$.

The measured intrinsic precision is about $8 \mu \mathrm{m}$ for the $R \Phi$ measurement while for $z$ it depends on the polar angle of the incident track, and goes from about $10 \mu \mathrm{m}$ for tracks perpendicular to the modules to $20 \mu \mathrm{m}$ for tracks with a polar angle of $25^{\circ}$. For charged particle tracks with hits in all three $R \Phi$ VD layers, the impact parameter resolution is $\sigma_{R \Phi}^{2}=\left[61 /\left(p \sin ^{3 / 2} \theta\right)\right]^{2}+20^{2} \mu \mathrm{m}^{2}$ while for tracks with hits in both the $R z$ layers it is $\sigma_{z}^{2}=\left[67 /\left(p \sin ^{5 / 2} \theta\right)\right]^{2}+33^{2} \mu \mathrm{m}^{2}$, where $p$ is the momentum in $\mathrm{GeV} / \mathrm{c}$.

\footnotetext{
${ }^{1}$ In the DELPHI cartesian coordinate system $z$ is along the beam line, $\Phi$ is the azimuthal angle in the $x y$ plane, $R$ is the radius and $\theta$ is the polar angle with respect to the $z$ axis.
} 


\section{Event Selection and Simulation}

The same event selection as in ref. [9] is used in this analysis. A charged particle is accepted with a polar angle between $20^{\circ}$ and $160^{\circ}$, a track length larger than $30 \mathrm{~cm}$, an impact parameter ${ }^{2}$ in the $R \Phi$ plane less than $2.5 \mathrm{~cm}$ and less than $10 \mathrm{~cm}$ in $z$ and a momentum larger than $200 \mathrm{MeV} / \mathrm{c}$. Neutral particles are accepted if the energy is larger than $700 \mathrm{MeV}$ in the barrel electromagnetic calorimeter HPC [7] and $400 \mathrm{MeV}$ in the forward calorimeter FEMC [7]. Neutral particles are used in the jet axis reconstruction and direction; the selection has been optimized to this extent. Events are selected if there are at least six charged particles and if the summed energy of the charged particles is larger than $15 \%$ of the center-of-mass energy. About 1,400,000 and 700,000 $Z$ events are selected respectively in the 1994 and 1995 data samples. The events collected at the three different center-of-mass energies (at the $Z$ peak and two energies approximately $1.8 \mathrm{GeV}$ above and below the resonant peak) are selected in this analysis.

Simulated events were generated using the JETSET 7.3 parton shower (PS) Monte Carlo program [10] tuned for the DELPHI data.

A good simulation of the impact parameter and the $b$-tagging variables for $Z$ decays into light quarks $(u d s c)$ is very important in this analysis. For this reason a fine tuning of the $R \Phi$ and $z$ impact parameter resolutions has been developed and applied [11].

\section{Analysis and results}

\subsection{The selection of $Z \rightarrow b \bar{b}$ events}

The $b$-tagging method used in this analysis is described in detail in ref.[12]. It combines, in a linear way, four different variables defined for each event hemisphere. The first variable $P_{H}^{+}$, originally proposed by ALEPH [13] and further developed by DELPHI [14], represents the probability that, in a given hemisphere, all the tracks with positive impact parameter originate from the primary vertex. The track impact parameters are computed separately in the $R \Phi$ plane and along the $z$ direction $[15,11]$. The sign of the impact parameter is defined with respect to the jet direction. It is positive if the point of closest approach of the track to the jet axis is downstream of the primary vertex along the jet direction, and negative if it is upstream. In this way the same sign is assigned to the $R \Phi$ and $z$ impact parameters. Additional selection variables are defined only for the event-hemispheres where a secondary vertex is reconstructed. They are: the effective mass, the rapidity with respect to the jet direction and the energy of the charged particles included in the secondary vertex. Reconstructed secondary vertices are accepted if $L / \sigma_{L} \geq 4$ where $L$ is the distance from the primary vertex and $\sigma_{L}$ is its uncertainty, which happens in about $55 \%$ of the hemispheres with $b$-quarks. Whenever a secondary vertex is reconstructed, the jet direction is recomputed as the direction from the primary vertex to the secondary vertex and the sign of the impact parameter is redefined accordingly [15].

For a given selection variable $x$ the ratio of the probability density function for background $f^{B}(x)$ and for signal events $f^{S}(x)$ is defined to be $y=f^{B}(x) / f^{S}(x)$ [12]. In the case of several independent variables the definition of this ratio is:

$$
y=\frac{f^{B}\left(x_{1}, \ldots x_{n}\right)}{f^{S}\left(x_{1}, \ldots x_{n}\right)}=\prod \frac{f^{B}\left(x_{i}\right)}{f^{S}\left(x_{i}\right)}=\prod y_{i} .
$$

\footnotetext{
${ }^{2}$ The impact parameter is defined as the distance of closest approach of a charged particle to the reconstructed primary vertex. The impact parameter in the $R \Phi$ and $R z$ planes are evaluated separately.
} 
The hemisphere is tagged as likely to contain a $b$ quark if the discriminating variable $y>y_{0}$; the choice of $y_{0}$ defines the efficiency and purity of the sample.

\subsection{The impact parameter analysis}

Events are divided into two hemispheres using the plane perpendicular to the thrust axis. One hemisphere of the event is used for $b$-tagging and the other to measure $n_{B}$. Long lived neutral particles like $K^{0}$ and $\Lambda$ are reconstructed and their decay products are excluded from this analysis. The efficiency of reconstructing $K^{0} \rightarrow \pi^{+} \pi^{-}$and $\Lambda \rightarrow p \pi^{-}$is about $36 \%$ and $30 \%$ respectively [7]. The ratio of the number of $K^{0}$ and $\Lambda$ reconstructed in data and simulation, when at least one of the tracks has hits in the VD, is $1.07 \pm 0.01$ and $1.15 \pm 0.02$ respectively.

Since the VD dominates the impact parameter resolution, only tracks with VD information are used. In particular, both for the probability computation and for the secondary vertex reconstruction, tracks are accepted only if they have at least one $R \Phi$ VD hit or at least one $R z$ VD hit [15]. Events are accepted if most of the tracks are inside the acceptance of the VD, i.e. if $\left|\cos \theta_{\text {thrust }}\right|<0.65$.

In order to reduce hemisphere-hemisphere correlations in the tagging efficiency for $b$-quarks, a separate primary vertex is computed for each hemisphere.

In the hemisphere opposite to the $b$-tagged one the difference:

$$
N_{+-}=\sum I P^{+}-\sum I P^{-}
$$

is computed, in which $\sum I P^{+}$is the number of tracks with positive impact parameter and $\sum I P^{-}$is the number of tracks with negative impact parameter. In figure 1 the mean value of $N_{+-}$is plotted for simulated events as a function of the discriminating cut in the tagging variable $y$ for primary $b, c$ and $u d s$ hadron decay products, while in figure 2 the same quantity is plotted for the data. The corresponding distribution obtained in the simulation has been superimposed as a histogram. The distribution of $N_{+-}$is well reproduced by the simulation over the whole range (figure $3 \mathrm{a}$ ). The measured quantity $N_{+-}$is lower than $n_{B}$ because of two reasons: (i) the request of having tracks with VD hits (they are about $74 \%$ of the total number of reconstructed tracks) and (ii) the experimental resolution that smears the jet direction.

Selecting events with a purity greater than $99.8 \%$ (i.e. for $y>3$ ) the average is:

$$
\begin{aligned}
& \left\langle N_{+-}\right\rangle=3.197 \pm 0.015 \quad(1994) \\
& \left\langle N_{+-}\right\rangle=3.157 \pm 0.021 \quad(1995)
\end{aligned}
$$

to be compared with:

$$
\begin{aligned}
& \left\langle N_{+-}^{M C}\right\rangle=3.177 \pm 0.012 \\
& \left\langle N_{+-}^{M C}\right\rangle=3.110 \pm 0.014
\end{aligned}
$$

in the simulation. The difference between 1994 and 1995 in the value of $\left\langle N_{+-}\right\rangle$is due to the difference in the VD alignment between the two years. The detector was aligned with about $10 \%$ better precision in 1994, resulting in about 0.5 more tracks per event with VD hits than in 1995 (see table 1).

If $N_{0}^{M C}$ is the generated value of $n_{B}$ in the simulation, the value of $n_{B}$ (and the corresponding error) in the data is determined from $N_{+-}$by the following equation:

$$
n_{B}=N_{0}^{M C}+\left(N_{+-}-N_{+-}^{M C}\right) \times S_{+-},
$$


where $S_{+-}=\frac{\Delta N_{0}^{M C}}{\Delta N_{+-}^{M C}}$ is the sensitivity of the analysis to $n_{B}$, with $\Delta N_{+-}^{M C}$ being the measured variation of $N_{+-}^{M C}$ for a change $\Delta N_{0}^{M C}$ of the generated $B$ multiplicity. In the simulation $N_{0}^{M C}=4.920 \pm 0.005$ (with the charmed hadron decay tracks included) [10]. The parameter $S_{+-}$of Eq.3 has been fitted by varying the generated multiplicity in the simulation by $\Delta N_{0}^{M C}= \pm 10 \%$, giving $S_{+-}=1.657 \pm 0.030$ and $1.631 \pm 0.036$ for the years 1994 and 1995 respectively. The maximum between the uncertainty from the fit and the difference of the values of $n_{B}$ obtained at $N_{0}^{M C^{\prime}}=N_{0}^{M C} \pm 10 \%$ is included as a systematic uncertainty below.

The values obtained are:

$$
\begin{aligned}
& n_{B}=4.953 \pm 0.032(\text { stat }) \\
& n_{B}=4.997 \pm 0.044(\text { stat })
\end{aligned}
$$

\subsection{Systematic uncertainties}

There are two sources of systematic uncertainties: detector effects and modelling of the $B$ hadron production and decays. This analysis shares many features with that of the $R_{b}$ measurement [9], and the evaluation of the systematic uncertainties has been done following the recommendations contained in ref. [16] and the work done in ref. [9].

The following contributions are considered and summarized in table 1.

Impact parameter sign assignment. The definition of the sign of the impact parameter is crucial to the tagging of $b$ events. The sign is defined with respect to the jet direction as explained in section 4.1. The jet direction is defined by the direction from the primary to the secondary vertex whenever a secondary vertex is reconstructed. It has been demonstrated that such a definition improves the b-tagging performance [15]. The analysis has been repeated by computing the sign of the impact parameter using for the jet direction the one defined by the jet clustering algorithm (JADE with $y_{\min }=0.01$ ). The measured value of $N_{+-}$is different with respect to the previous one, but remains in agreement with the value obtained in the simulation:

$$
\begin{aligned}
& <N_{+-}^{\prime}>=2.873 \pm 0.015 \\
& \left.<N_{+-}^{\prime M C}\right\rangle=2.855 \pm 0.012
\end{aligned}
$$

for 1994 data. Similarly for 1995 :

$$
\begin{aligned}
& <N_{+-}^{\prime}>=2.843 \pm 0.020 \\
& \left\langle N_{+-}^{\prime M C}\right\rangle=2.797 \pm 0.016 .
\end{aligned}
$$

Using equation 3 , the measured values are $n_{B}=4.955 \pm 0.038$ and $5.009 \pm 0.049$ for the years 1994 and 1995 respectively (with $S_{+-}^{\prime M C}=1.968 \pm 0.032$ and $1.926 \pm 0.044$ ).

Detector resolution effects. To estimate the effects of the detector resolution, two different tests have been done. First, the simulation has been rerun with a tuning of the $R \Phi$ and $z$ impact parameter resolutions [11], showing a worse agreement with the data than the default one (about $4 \%$ relative difference in the light and charm quark efficiencies). Second, the resolution of the detector as estimated from the data has been used in the definition of the tagging probability of simulated events. This second test is sensitive to systematics related to the simulation of the charm background since charmed particles have a detectable lifetime and a non zero charged decay multiplicity (see figure 1).

Radial misalignment of the vertex detector could shift the central Gaussian part of the impact parameter distribution and shift its mean from zero. The systematic error 
due to this effect has been estimated by shifting the distribution of the impact parameter in the simulated sample by the difference of data from simulation $(0.2 \mu \mathrm{m})$.

Tracking Efficiency. The tracking efficiency was computed in the simulation by comparing the number of reconstructed tracks and generated charged particles. Applying the same track selection as used in the analysis, the tracking efficiency was found to be $0.989 \pm 0.001$. The $1 \%$ loss was considered as the systematic error on $n_{B}$. The possible loss of tracks, not properly simulated, in the cracks between the TPC sectors has been studied. The difference between the number of tracks in data and simulation, integrated over the regions of good and bad acceptance of the TPC, has been found to be about $0.2 \%$. The eventual loss in efficiency due to the requirement to have VD hits in the tracks has been evaluated by computing the ratio between the number of tracks in data and in simulation with VD hits in an anti- $b$-tagged sample. It has been found to be 0.995 .

Another method to study the tracking efficiency is to extract the average charged particle multiplicity for the total $Z$ sample using the selected tracks (i.e. with VD hits). If $N_{V D}$ and $N_{V D}^{M C}$ is the mean value of charged tracks with VD hits in data and simulation respectively and $N_{Z^{0}}$ is the generated value in the simulation (JETSET 7.3 [10]), the average charged particle multiplicity for the total $Z$ is given by $N_{V D} \times N_{Z^{0}} / N_{V D}^{M C}$. The values were measured to be $21.062 \pm 0.008$ (20.96 \pm 0.01$)$ for 1994 (1995); the errors are statistical only. Such results are in good agreement with the world average of $21.00 \pm 0.13$ [17]. From this method an error of $0.6 \%$ is assigned to the multiplicity scale uncertainty.

The two methods give consistent results; to be conservative, the largest uncertainty from the first method has been chosen.

To study the effect due to the loss of low momentum particles the following procedure is used. All the particles in one hemisphere are boosted back to the reconstructed $B$ center-of-mass system and the quantity $N_{+-}$is computed as a function of $\cos \left(\theta_{B}\right)$, where $\theta_{B}$ is the angle between the particle and the $B$ direction in the $B$ rest frame. The low momentum particles emitted backwards can be lost in the reconstruction. The maximum difference, $0.5 \%$, between the ratios $\left\langle N_{+-}\right\rangle /\left\langle N_{+-}^{M C}\right\rangle$ in four $\cos \left(\theta_{B}\right)$ bins is the systematic uncertainty due to the loss of low momentum particles. This contribution is already included in the tracking efficiency uncertainty.

Hemisphere correlation. The influence of the hemisphere correlation on the analysis can be studied by doing the measurement with a single fitted primary vertex per event. It was estimated in ref.[9] that the major contribution to the hemisphere correlation in the tagging efficiency for $b$-quarks comes from the common primary vertex. The correlation can be substantially reduced whenever a separate primary vertex is computed for each hemisphere. The difference between the values of $n_{B}$ computed using a single primary vertex per event and a separate primary vertex per hemisphere is given as the systematic uncertainty due to the hemisphere correlation. It is 0.015 and 0.034 for 1994 and 1995 years respectively (see table 1 ).

The linearity of the measurement was verified by varying the average multiplicity of $B$ decays in the simulation by $\pm 10 \%$.

In addition, since for the second analysis (see section 4.5 ) the shape of the multiplicity distribution had to be varied (see figures $3 \mathrm{~b}$ and $3 \mathrm{c}$ ); the difference between the two estimated values has been taken as the systematic uncertainty from this source.

The average $b$ lifetime was varied as recommended in ref. [16].

The gluon splitting rates into $b \bar{b}$ and into $c \bar{c}$ were varied as recommended in ref. [16].

The branching ratio of $\mathbf{D}$ mesons into kaons was varied by $\pm 15 \%$. As for the measurement of $R_{b}$ (see ref. [9]), this is the largest error, coming from uncertainties in charm hadron decay properties. 
The $K^{0}$ and $\Lambda$ production rates in the simulation of $b \bar{b}$ events were varied by $\pm 10 \%$ and $\pm 20 \%$ respectively with respect to the measured values [4].

All studied systematic errors are summarized in table 1.

\begin{tabular}{||l|c|c|c||}
\hline Source of systematics & Range & $\Delta n_{B} \times 10^{-2}$ & Corr. \\
\hline Sign assignment & & $\pm 0.2(1.2)$ & 0 \\
Detector resolution & & $\pm 2.6(3.1)$ & 0 \\
Radial VD alignment & & $\pm 1.4(2.0)$ & 0 \\
Tracking efficiency & & $\pm 5.0(5.0)$ & 1 \\
Hemisphere correlations & & $\pm 1.5(3.4)$ & 0 \\
Linearity & $\pm 10 \%$ & $\pm 0.1(0.7)$ & 0 \\
Shape correction & & $\pm 1.7(0.8)$ & 0 \\
$B$ lifetime & $1.55 \pm 0.05 \mathrm{ps}$ & \pm 1.9 & 1 \\
Gluon splitting $g \rightarrow b \bar{b}$ & $(0.31 \pm 0.11) \%$ & \pm 0.4 & 1 \\
Gluon splitting $g \rightarrow c \bar{c}$ & $(2.38 \pm 0.48) \%$ & \pm 0.4 & 1 \\
$B R\left(D \rightarrow K^{0} X\right)$ & $0.46 \pm 0.06$ & \pm 1.1 & 1 \\
$K^{0} \Lambda$ production rate & $\pm 10 \%, \pm 20 \%$ & \pm 1.1 & 1 \\
\hline Total & & $\pm 6.7(7.6)$ & \\
\hline
\end{tabular}

Table 1: Systematic errors on the measurement of $n_{B}$ with the impact parameter method for 1994 (1995) data. The last column shows the correlation coefficient between years.

\subsection{Results}

The final result for 1994 data is:

$$
n_{B}=4.953 \pm 0.032 \pm 0.067
$$

and for 1995 data is

$$
n_{B}=4.997 \pm 0.044 \pm 0.076,
$$

where the first error is statistical and the second includes all systematic effects.

The results are compatible and have been combined assuming independent statistical errors. The systematic error has been computed considering the full correlation of the errors due to the method and to sample composition and the independence of the detector related systematics because of the separate alignment and tuning procedure [11] for the two years. The correlation coefficient assumed between the two data sets is shown in the last column of table 1.

With these assumptions the result for the combined 1994 and 1995 data samples is:

$$
n_{B}=4.968 \pm 0.026(\text { stat }) \pm 0.064(\text { syst }) .
$$

\subsection{A cross check: the Secondary Vertex Analysis}

An alternative method used to estimate the charged particle multiplicity is to count the tracks that are assigned to a secondary vertex. A secondary vertex is reconstructed requiring at least two tracks with $V D$ hits and requiring the fit $\chi^{2} \leq 4$. It is accepted if $L / \sigma_{L}>4$, where $L$ is the distance from the primary vertex and $\sigma_{L}$ is its uncertainty. The tracks that are (i) excluded by the secondary vertex search but (ii) pass close to the 
estimated flight direction of the $B$ hadron and (iii) are far from the primary vertex are forced to belong to the secondary vertex. This takes into account the cases where a $D$ meson decays far from the $B$ decay vertex. Since a secondary vertex is reconstructed with at least two tracks, the multiplicity distribution will be biased towards higher values.

The distributions of the tracks retained at the secondary vertex $N_{s e c}$ in data and simulation do not agree at high values, as shown in figure $3 \mathrm{~b}$. Reweighting the simulation events in order to narrow the generated multiplicity distribution by about $5 \%$ gives better agreement, as shown in figure $3 \mathrm{c}$; the results given below use the reweighted simulation. Counting the number of tracks retained at the secondary vertex, $N_{s e c}$, as a function of the $b$-tagging variable (i.e. for increasing purity), (see figure 4), the following results have been obtained, when selecting events with a $b$ purity greater than $99.8 \%$ (i.e. $y>3$ ), for the year 1994:

$$
\begin{aligned}
<N_{\text {sec }}> & =4.570 \pm 0.011 \\
<N_{\text {sec }}^{M C}> & =4.583 \pm 0.009
\end{aligned}
$$

and for the year 1995:

$$
\begin{aligned}
& <N_{s e c}>=4.580 \pm 0.016, \\
& <N_{s e c}^{M C}>=4.604 \pm 0.013 .
\end{aligned}
$$

If $N_{0}^{M C}$ is the generated value in the simulation, the value of $n_{B}$ is determined as in the previous method:

$$
n_{B}=N_{0}^{M C}+\left(N_{s e c}-N_{s e c}^{M C}\right) \times S_{s e c},
$$

where the various terms have the same meaning as in Eq. 3 with the index 'sec' used to identify the alternate analysis method. The coefficients of Eq. 4 have been determined by varying the generated multiplicity in the simulation by $\Delta N_{0}^{M C}= \pm 10 \%$, giving $S_{s e c}^{M C}=$ $1.923 \pm 0.029$ and $1.905 \pm 0.041$ for the 1994 and 1995 data sets respectively. The maximum between the uncertainty from the fit and the difference of the values of $n_{B}$ obtained at $N_{0}^{M C^{\prime}}=N_{0}^{M C} \pm 10 \%$ is included as a systematic uncertainty below.

The same systematic errors considered in the first analysis have been evaluated and are listed in table 2. It should be noticed that this method suffers from a bias because of the requirement to have at least 2 tracks belonging to the secondary vertex. This translates into a reduced sensitivity since a variation of $10 \%$ on the generated multiplicity gives a variation of only $6 \%$ on the measured one. Moreover the shape of the $N_{s e c}$ distribution does not agree between data and simulation and the simulation had to be reweighted in order to reach agreement. The difference between the results obtained with and without reweighting the simulation is given as a systematic error. For these reasons this analysis is used only as a cross check and its results are not combined with the previous analysis.

The values of $n_{B}$ obtained with this method are:

$$
\begin{gathered}
n_{B}=4.895 \pm 0.029(\text { stat. }) \pm 0.108(\text { syst. })(1994) \\
n_{B}=4.874 \pm 0.039(\text { stat. }) \pm 0.118(\text { syst. })(1995) .
\end{gathered}
$$

Assuming correlation coefficients as described in 4.4, combining the two years yields:

$$
n_{B}=4.888 \pm 0.023 \text { (stat.) } \pm 0.104 \text { (syst.). }
$$

This result is compatible with the one obtained with the impact parameter analysis, although it suffers from a larger systematic uncertainty. 


\begin{tabular}{||l|c|c|c||}
\hline Source of systematics & Range & $\Delta n_{B} \times 10^{-2}$ & Corr. \\
\hline Detector resolution & & $\pm 1.9(2.5)$ & 0 \\
Radial VD alignment & & $\pm 0.4(0.4)$ & 0 \\
Tracking efficiency & & $\pm 4.8(3.7)$ & 0 \\
Hemisphere correlations & & $\pm 2.3(4.6)$ & 0 \\
Linearity & $\pm 10 \%$ & $\pm 5.0(6.0)$ & 0 \\
Shape correction & $1.55 \pm 0.05 \mathrm{ps}$ & \pm 0.3 & 1 \\
$B$ lifetime & $(0.31 \pm 0.11) \%$ & \pm 0.1 & 1 \\
Gluon splitting $g \rightarrow b \bar{b}$ & $(2.38 \pm 0.48) \%$ & \pm 0.1 & 1 \\
Gluon splitting $g \rightarrow c \bar{c}$ & $0.46 \pm 0.06$ & \pm 5.3 & 1 \\
$B R\left(D \rightarrow K^{0} X\right)$ & $\pm 10 \%, \pm 20 \%$ & \pm 1.4 & 1 \\
$K^{0} \Lambda$ production rate & & $\pm 10.8(11.8)$ & \\
\hline Total
\end{tabular}

Table 2: Systematic errors on the measurement of $n_{B}$ with the secondary vertex method for 1994 (1995) data. The last column shows the correlation coefficient between years.

\section{Summary}

The average charged decay multiplicity $n_{B}$ of weakly decaying $B$ hadrons produced in a $b$-quark jet at the $Z$ pole energy was measured by counting the difference between the tracks with positive and negative impact parameters in a hemisphere:

$$
n_{B}=4.97 \pm 0.03 \text { (stat.) } \pm 0.06 \text { (syst.) },
$$

where the $K^{0}$ and $\Lambda$ decay contributions were not considered. A second method that counts the tracks pointing to a secondary vertex gave a consistent result with a larger systematic uncertainty.

At the $\Upsilon(4 S)$ CLEO [1] and ARGUS [2] measured respectively $5.15 \pm 0.03 \pm 0.15$ and $5.04 \pm 0.03 \pm 0.12$, while at LEP in previous analyses OPAL [3], DELPHI [4] and L3 [5] measured $n_{B}=5.03 \pm 0.04 \pm 0.49, n_{B}=5.33 \pm 0.04 \pm 0.38$ and $n_{B}=4.90 \pm 0.04 \pm 0.10$ respectively. In all the cases $K^{0}$ and $\Lambda$ decay products had been subtracted. In the DELPHI result [4], the contribution from $B^{* *} \rightarrow \pi^{ \pm} B$, which is measured to be $0.16 \pm 0.03$ [18], is subtracted as well. Taking these facts into account, the present measurement is consistent with the previous ones, but has a smaller uncertainty.

\section{Acknowledgements}

We are greatly indebted to our technical collaborators and to the funding agencies for their support in building and operating the DELPHI detector, and to the members of the CERN-SL Division for the excellent performance of the LEP collider.

We are also grateful to the technical and engineering staffs in our laboratories and we acknowledge the support of

Austrian Federal Ministry of Science, Research and Arts,

FNRS-FWO, Belgium,

FINEP, CNPq, CAPES, FUJB and FAPERJ, Brazil,

Czech Ministry of Industry and Trade, GA CR 202/96/0450 and GA AVCR A1010521,

Danish Natural Research Council, 
Commission of the European Communities (DG XII),

Direction des Sciences de la Matière, CEA, France,

Bundesministerium für Bildung, Wissenschaft, Forschung und Technologie, Germany,

General Secretariat for Research and Technology, Greece,

National Science Foundation (NWO) and Foundation for Research on Matter (FOM),

The Netherlands,

Norwegian Research Council,

State Committee for Scientific Research, Poland, 2P03B00108, 2P03B03311 and 628/E78-SPUB-P03-023/97,

JNICT-Junta Nacional de Investigação Científica e Tecnológica, Portugal,

Vedecka grantova agentura MS SR, Slovakia, Nr. 95/5195/134,

Ministry of Science and Technology of the Republic of Slovenia,

CICYT, Spain, AEN96-1661 and AEN96-1681,

The Swedish Natural Science Research Council,

Particle Physics and Astronomy Research Council, UK,

Department of Energy, USA, DE-FG02-94ER40817. 


\section{References}

[1] CLEO Collaboration, R. Giles et al., Phys. Rev. D30 (1984), 2279.

[2] ARGUS Collaboration, H. Albrecht et al., Z. Phys. C54 (1992), 20.

[3] OPAL Collaboration, R. Akers et al., Z.Phys C61 (1994) 209.

[4] DELPHI Collaboration, P. Abreu et al., Phys. Lett B347 (1995) 447.

[5] L3 Collaboration, M. Acciari et al., Phys. Lett. B416 (1998) 220.

[6] V. Chabaud et al., Nucl. Inst. Meth A368 (1996) 314.

[7] DELPHI Collaboration, P. Abreu et al., Nucl. Inst. Meth. A378 (1996) 57.

[8] N. Bingefors et al., Nucl. Inst. Meth. A328 (1993) 447.

[9] DELPHI Collaboration, P. Abreu et al., Z. Phys. C70 (1996) 531;

DELPHI Collaboration, P. Abreu et al., Z. Phys. C66 (1995) 323.

[10] T. Sjöstrand et al., in "Z physics at LEP 1", CERN 89-08, CERN, Geneva, 1989; Comp. Phys. Comm. 39 (1986) 347.

[11] G. Borisov and C. Mariotti, Nucl. Inst. Meth. A372 (1996) 181.

G. Borisov and C. Mariotti, Tuning of the Track Impact Parameter Resolution of the Upgraded DELPHI Detector, ISS-INFN 97/14 (also DELPHI note, DELPHI 97-95 PHYS 717).

[12] G. Borisov, Combined b-tagging, CEA/SACLAY DAPNIA/SPP 97-28 (also DELPHI note, DELPHI 97-94 PHYS 716.)

[13] ALEPH Collaboration, D. Buskulic et al., Phys. Lett. B313 (1993) 535.

[14] G.V. Borisov, Lifetime Tag of Events with B-hadrons with the DELPHI Detector, preprint IHEP (Protvino), 94-98 (1994).

[15] G.V. Borisov and C. Mariotti, Performance of b-tagging in DELPHI at LEP2, preprint CEN SACLAY DAPNIA/SPP-97-06 and INFN-ISS 97/3, April 1997.

[16] The LEP Electroweak Working Group, LEPHF/96-01.

[17] R.M. Barnett et al. (Particle Data Group), Phys. Rev. D54 (1996) 1 and 1997 offyear partial update for the 1998 edition available on the PDG WWW pages (URL: http://pdg.lbl.gov/).

[18] ALEPH Collaboration, D. Buskulic et al., Z. Phys. C69 (1996) 393. 


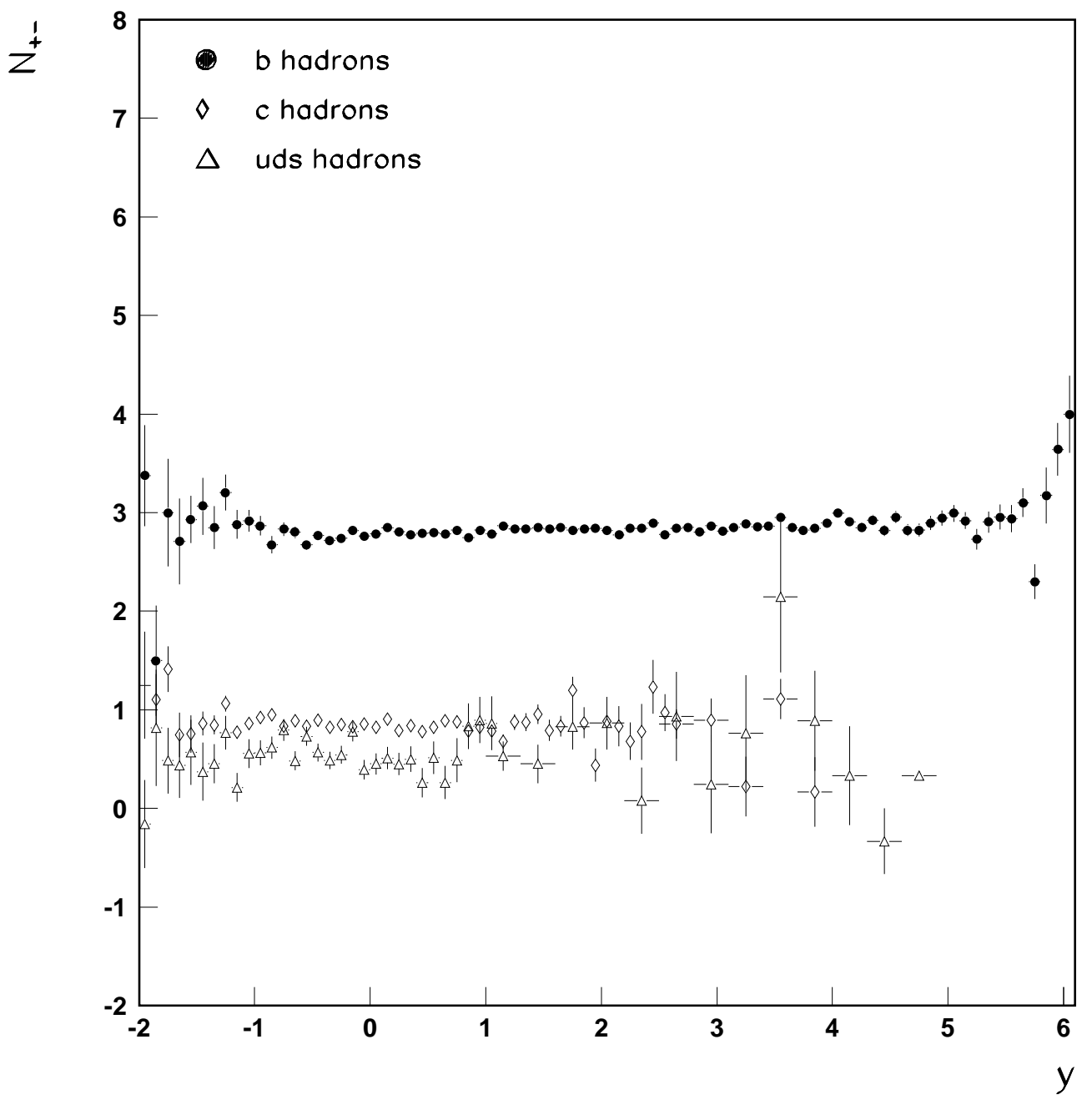

Figure 1: The average value of $N_{+-}$(the difference between the number of tracks with positive and negative impact parameter in the hemisphere opposite to the b-tagged one) from simulation is shown for $b, c$ and uds hadron decay products as a function of the discriminating cut in the tagging variable $y$, i.e. for increasing $b$ purity in the selected sample of events. The cut used in this analysis is $y>3$. which corresponds to a $b$ purity $>99.8 \%$. 


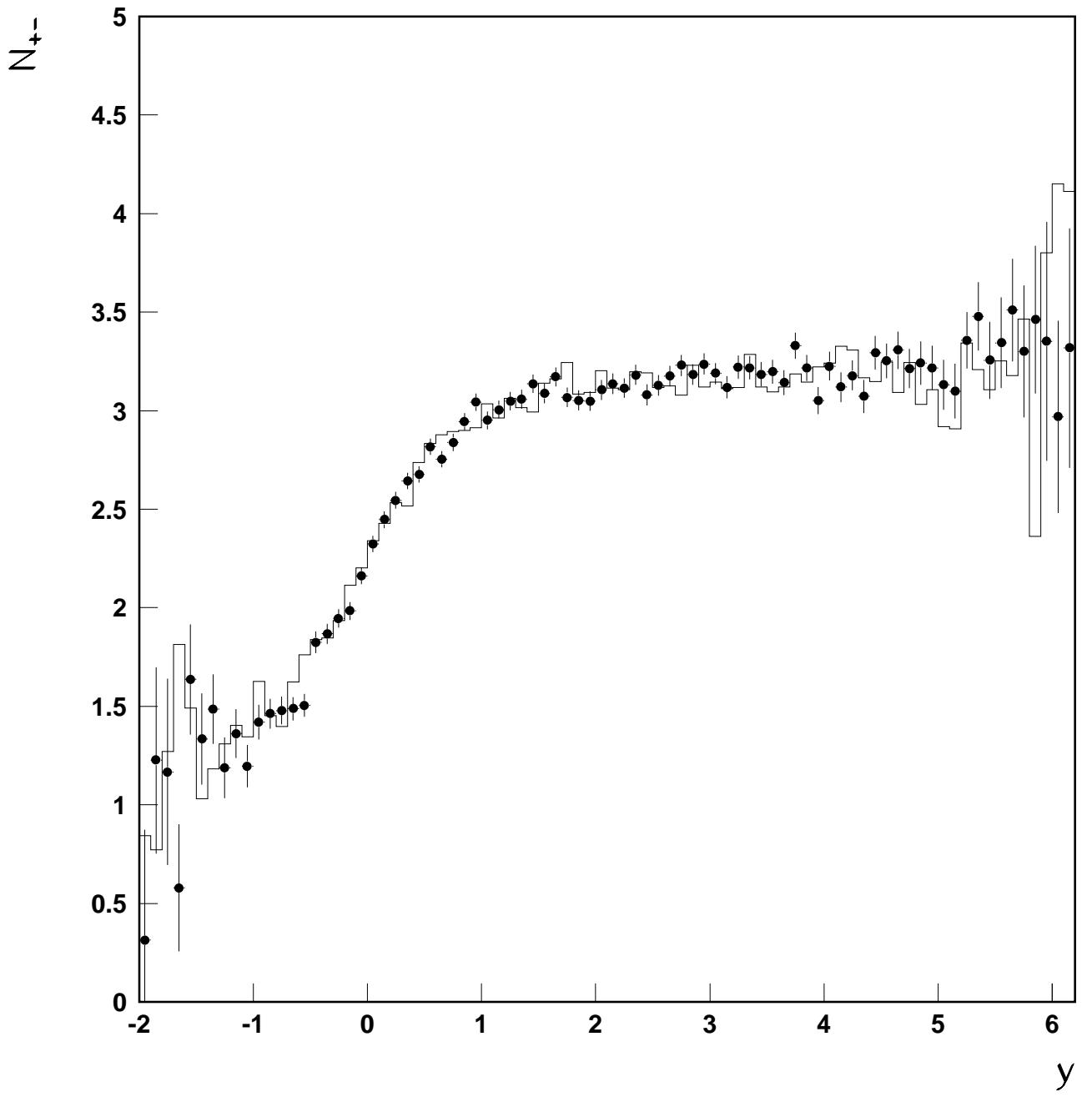

Figure 2: Average value of $N_{+-}$(the difference between the number of tracks with positive and negative impact parameter in the hemisphere opposite to the b-tagged one) for the data collected in 1994 and 1995 (full points). The superimposed histogram shows the result of the simulation. 

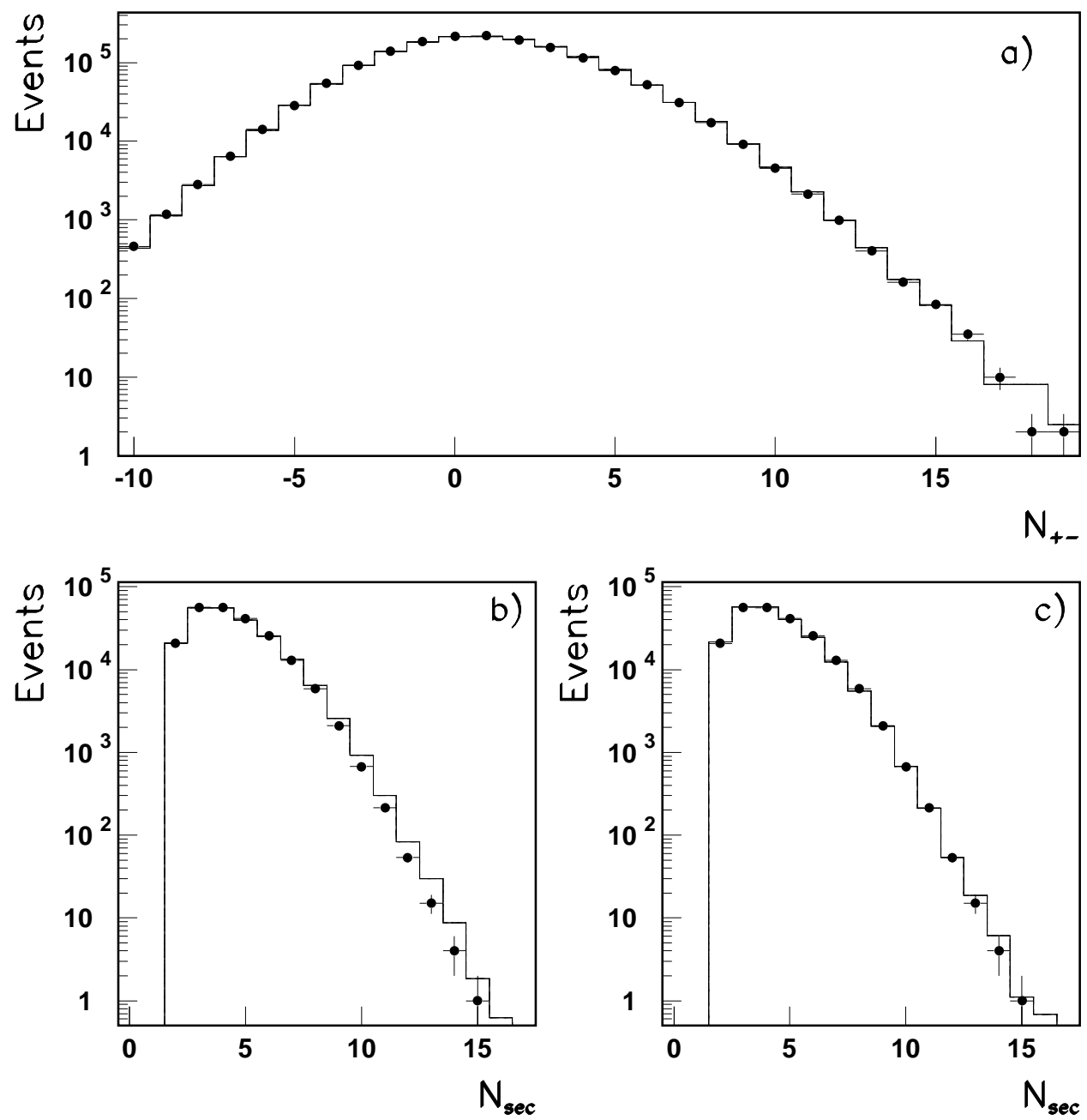

Figure 3: (a) The distribution of $N_{+-}$is shown for real data (dots) and simulation (histogram, normalized to the real data). The distribution of $N_{s e c}$ is shown for real data (dots) and simulation (histogram) (b) before and (c) after changing the shape of the multiplicity distribution in the simulation. 


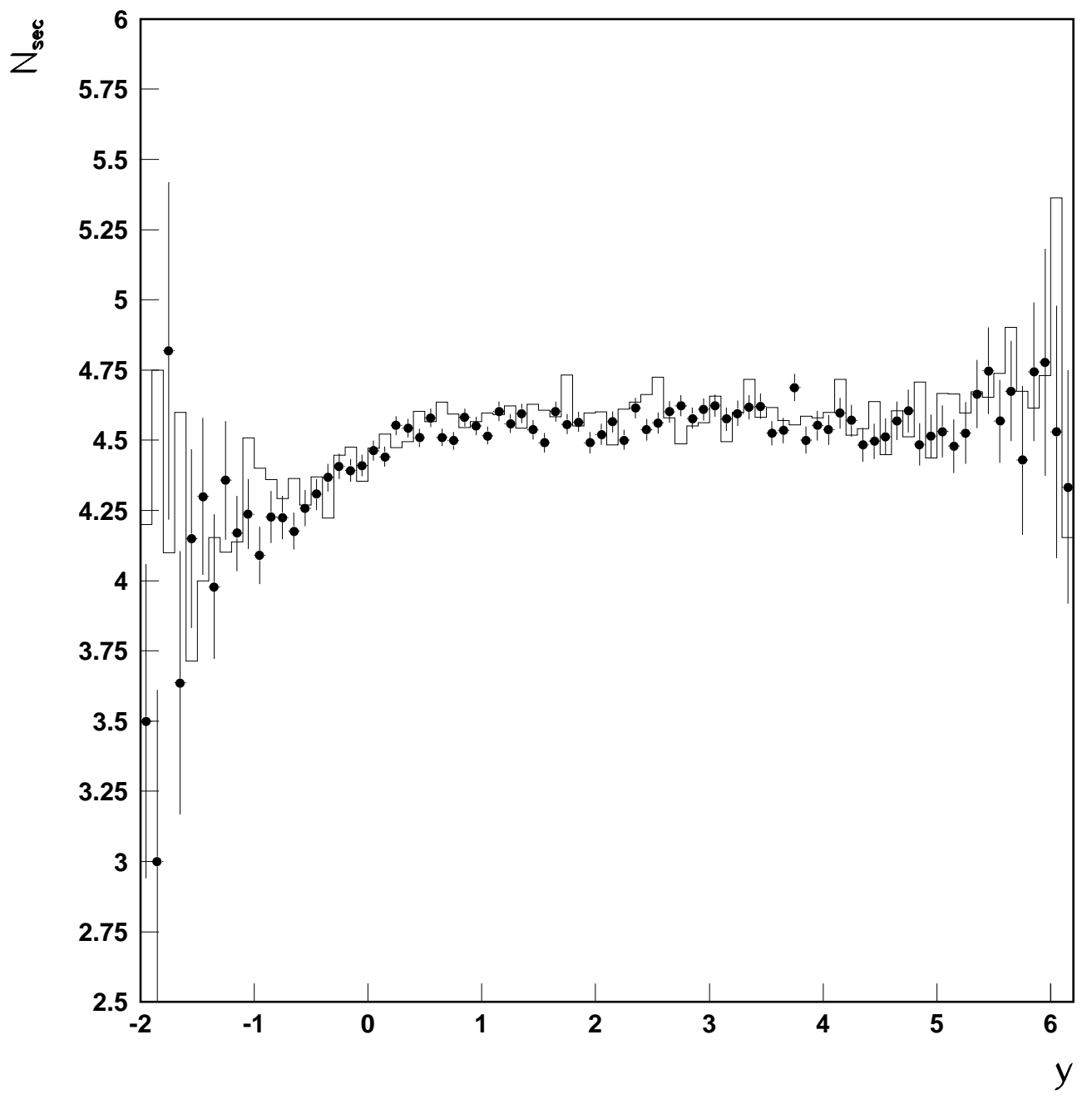

Figure 4: The value of $N_{s e c}$ is shown for the data collected in 1994 and 1995 (full points). The superimposed histogram is the simulation. 